\title{
Teaching safe prescribing to medical students: perspectives in the UK
}

This article was published in the following Dove Press journal:

Advances in Medical Education and Practice

17 April 2015

Number of times this article has been viewed

\author{
Hamde Nazar' \\ Mahdi Nazar ${ }^{2}$ \\ Charlotte Rothwell' \\ Jane Portlock ${ }^{3}$ \\ Andrew Chaytor' \\ Andrew Husband' \\ 'School of Medicine, Pharmacy \\ and Health, Durham University, \\ UK; ${ }^{2}$ Cumberland Infirmary, North \\ Cumbria University Hospitals NHS \\ Trust, UK; ${ }^{3}$ School of Pharmacy and \\ Biomedical Sciences, University of \\ Portsmouth, UK
}

\begin{abstract}
Prescribing is a characteristic role of a medical practitioner. On graduating from medical school, students are presumed to have acquired the necessary pharmacology knowledge underpinning the therapeutics and developed their personal skills and behaviors in order to write a safe and effective prescription (The Four Ps). However, there are reports of errors in medical prescribing and dissatisfied feedback from recent graduates, which evidence potential flaws in the current training in the practice of prescribing. We examine the Four Ps from a systems approach and offer scope for educators and curriculum designers to review and reflect on their current undergraduate teaching, learning, and assessment strategies in a similar manner. We also adopt a national framework of common competencies required of all prescribers to remain effective and safe in their area of practice as a more objective layer to the broader learning outcomes of the General Medical Council Tomorrow's Doctors 2009. This exercise demonstrates where standard, recognized competencies for safe prescribing can be accommodated pedagogically within existing medical curricula.
\end{abstract}

Keywords: prescribing, medical curriculum, clinical pharmacology teaching, therapeutics, education

\section{Introduction}

"Most doctors are prisoners of their education and shackled by their profession."1 Upon graduation from a medical school in the UK, students are expected to have formed into the optimal scholar and scientist, practitioner, and professional, having met all outcomes as stipulated in the General Medical Council (GMC) Tomorrow's Doctors 2009. ${ }^{2}$ Medical curricula must be constructed to deliver and provide the necessary background and technical skills, learning opportunities and environments, and robust assessment strategies to ensure that students are appropriately equipped and prepared for practice. The "prison" that is established via their education should be one based on secure scientific foundation, supporting walls of complex clinical application, and levels of high personal and professional values so as to uphold the safety and integrity of patient-centered care. However, studies exist, and are still being produced, that report upon the frequency of prescribing errors, which contribute to one of the most common adverse events in health care. ${ }^{3-6}$ Reported errors are made by all grades of doctor, although less commonly by consultants $(5.9 \%){ }^{7}{ }^{7}$ notably foundation year 1 (FY1), and foundation year 2 (FY2) are acknowledged to be at high risk of contributing to the error rates in particular cohorts, ${ }^{8,9} 8.4 \%$ and $10.3 \%$, respectively. ${ }^{7}$ Compounding this further is the fact that $75 \%$ of all inpatient prescriptions are written
Correspondence: Hamde Nazar School of Medicine, Pharmacy and Health, Durham University, Queen's Campus, Wolfson Institute, Stockton-on-Tees, TSI 7 6BH, UK

Tel +44 I9I 3340250

Email hamde.nazar@durham.ac.uk 
by recent graduates. ${ }^{6}$ The factors contributing to prescribing errors are extremely complex and include 1) characteristics of the clinical environment, 2) prescribing culture, and 3) workload, time, and support. ${ }^{10}$ However, at an individual level, prescribing errors are attributable to a lack of training in practical prescribing and failure to link theory and practice. ${ }^{7}$ This situation directs attention to the current undergraduate curriculum, which does not appear to be preparing trainee doctors as well as it should be. ${ }^{11,12}$ In fact, it is falling short of developing and nurturing the knowledge, skills, and behavior for graduates to "Prescribe drugs safely, effectively and economically" as stipulated by the specific GMC Tomorrow's Doctors outcome. ${ }^{2}$

There has been much academic debate around the educational tools and interventions to create the appropriate teaching, learning, and assessment strategies to ensure this GMC outcome is achieved. ${ }^{3}$ Published work reports on these strategies as potential improvements to remedy the error rate as well as to combat the reported lack of contextual knowledge and dissatisfaction with training as expressed by recent graduates with respect to prescribing. ${ }^{3,13-17}$ The vast majority of research tends to report on initiatives that are isolated teaching or training opportunities within the curriculum or simplistic in structure, which does not truly reflect the complexity of the practice of prescribing in context. ${ }^{14,18-20}$

There has been the development of a single-competency framework for all prescribers by the National Prescribing Centre (NPC) commissioned by the Department of Health. ${ }^{21}$ This national body has recognized, over iterations of developing prescribing competency frameworks for each of the nonmedical prescribing professions, that a common set of competencies underpin prescribing regardless of professional background. This framework represents an outline of those competencies that, if acquired and maintained, can aid all professionals to become and remain effective prescribers in their area of practice. ${ }^{21}$ Competencies can be described as a combination of knowledge, skills, motives, and personal traits, which in turn can be related to performance. The development of competencies should help individuals improve their performance and work more effectively. ${ }^{22}$ Here, we have adopted this competency framework and proposed a mapping of this to the GMC standards. The further layer of competency contributed by the framework provides more objective, measurable learning outcomes to the GMC broad statements. The aim is to provide a tool for curriculum developers to review and assess their current teaching and learning strategies and appreciate where and how elements of safe and effective prescribing competencies can be built into the curriculum. In including these competencies through the educational program, medical schools have the opportunity to evidence that students have been taught within a robust structure that meets nationally stipulated prescribing recommendations. However, we have to acknowledge the existing flaw in equating competencies to performance and assessing on this basis, since competencies tend to be behaviors and actions carried out in controlled representations of professional practice, and performance describes practice in the actual clinical setting. ${ }^{23}$ So considering this, we hope to advocate that these taught competencies would be assessed by their performance in real-life settings where feasible.

We will source and review extensive primary and secondary research, and supplement with knowledge imparted from gray literature. The aim is to examine and provide some critique on the key messages relating to the practice of prescribing that resonates collectively within the literature and provide supporting evidence through relevant studies. This will disentangle the educational components that facilitate the teaching and learning of safe and rational prescribing and inform educators of the holistic approach required when considering reforming curricula. The ultimate intention is to help to address the deficits in the prescribing competencies of their graduates.

\section{Method}

Our search strategy identified research on medical curriculum in the UK that were published after the updated version of the revolutionary GMC Tomorrow's Doctors 1993 recommendations, ie, 2003. However, due to the more recent recommendations in 2009, most research contained in this review was found in studies carried out after this latter publication. The following electronic databases were searched: MEDLINE, EMBASE, PubMed, SCOPUS, and Web of Science. This was supplemented with a website and gray literature search to obtain further reports, informed specialist commentaries, conference proceedings, and professional journal and articles. Papers were excluded if they were in non-English language, related to other health care professional (eg, nurse, pharmacy) education, or published before 2003. Two authors independently carried out the overarching searches and analyzed for emerging themes. These were discussed on an iterative basis in consultation with a third author to correct any discrepancies. The themes were refined through discussion with all authors to generate key messages, which are based around the Four Ps. 
Themes derived from the articles are as follows:

Prescribing is not about the isolated pharmacology but it requires contextualization with a patient;

Prescribing is not about the final prescription but the underlying process;

Prescribing is not only about personal knowledge, skill, and attitude but is also a participative process;

Prescribing should not be presumed, it should be appropriately proven.

A systems approach was adopted to discuss these themes. This method provides a way to selectively handle the detail that may complicate thinking in a transparent manner, in order to reveal the underlying features of a situation from a set of explicit perspectives. ${ }^{24}$ For example, a systems approach to pharmacology is based on the understanding that, if one considers the concept as a whole, ie, drug action in a patient with a disease(s) at a particular dose by a specific route, rather than focusing on its component parts, ie, isolated drug action, then there are considerations required that would not apply if considering the component parts. Another aspect of the systems approach is that the situation is considered with its complexity and uncertainty, and part of the problem is to establish and agree what the problem is, and it is acknowledged that there will rarely be a single "right" solution. In line with this, our approach is of problem situation and of resolution (improving the situation) rather than solution (solving the problem). ${ }^{24}$

\section{Findings}

\section{Prescribing is not about the isolated pharmacology but it requires contextualization with a patient}

There has been ongoing debate as to the contributing factors behind the reported lack of prescribing competencies of recent graduates. One argument holds the recommendations of GMC Tomorrow's Doctors 1993 accountable. The subsequent redesign in medical curriculum as a result of this document stripped away teaching in silos and introduced the integration of scientific theory with clinical practice. ${ }^{5,25}$ This saw the "disappearance" of formal disparate clinical pharmacology and therapeutics (CPT) teaching that tackled generic issues relating to the use of drugs in a variety of clinical settings. ${ }^{26}$ The suggested weakened exposure of students to recognizable CPT material has been acknowledged to coincide with the rise in concern about the extent to which graduates are prepared to assume their responsibilities as prescribers. ${ }^{15,27}$
However, the O'Shaughnessy study ${ }^{28}$ found that, contrary to the perceived demise of CPT, a large number of UK medical schools reported to have a dedicated CPT course integrated, albeit generally more vertically than horizontally, within their medical curricula. ${ }^{28}$ Also, when evaluating the reasons for medication errors in practice, it is often not simply due to deficits in core underlying knowledge. ${ }^{29}$ Tully et al grouped causes according to Reason's model of accident causation into active failures, error-provoking conditions, and latent conditions, demonstrating the myriad of interacting factors involved in the complexity of prescribing within the context of a patient and the clinical environment. ${ }^{10}$ Indeed, it is acknowledged that tests of knowledge alone are insufficient to properly assess the educational intervention's impact on prescribing. ${ }^{20,30-32}$

Richir et $\mathrm{al}^{33}$ advocate that the core knowledge, skills, and attitudes of clinical pharmacology must be gained simultaneously so that a student is able to rationalize therapeutic strategies. A suggested curriculum could be structured so that the knowledge of pharmacology is acquired alongside the development of the skills for applying that knowledge in the therapeutic management of a patient. This should support the development from acquisition of knowledge and skills to the behavior of prescribing in clinical practice. This context learning has been demonstrated to be more effective than sequential learning, where the learning and the application of knowledge are separate, and is evidenced in theories from cognitive psychology and medical problem solving. ${ }^{33-35}$ An integrated curriculum is therefore the optimal environment for CPT delivery. Maxwell et al ${ }^{26,36-38}$ have contributed to various research that recommends how to operationalize this within medical schools, particularly in the British Pharmacological Society (BPS) curriculum for clinical pharmacology. Maxwell et $\mathrm{al}^{38}$ first reported on the development of an online resource in 2006, which would be continually accessible to students throughout their medical studies. The dynamic package was developed to hold a core drug list with related fundamental pharmacology, clinical pharmacology, therapeutics and toxicology. It links to lecture notes, PowerPoint presentations, computer-assisted learning packages, and external sources of information; for example, the British National Formulary (BNF) was also built in. This strategy raised the profile of the previously "invisible" CPT within the curriculum, but also ensured, with continual referral and linking across modules, that the theme was delivered and accessed horizontally and vertically throughout the program. ${ }^{38}$ The subsequent BPS curriculum promotes that, 
indeed, the CPT should be organized to be visible, vertical, and horizontal, building upon the basics of pharmacology, onto clinical pharmacology and therapeutics and culminating with an emphasis on practical prescribing so that students are always able to integrate scientific fact into rational practice around a patient. ${ }^{37}$ The visibility of the expected outcome of safe and effective prescribing should be in place from the very beginning of the teaching-learning process. According to Galperin, ${ }^{39}$ this initial awareness will help students develop their independent learning processes though their own activities in order to achieve the required action or outcome. Presenting the required knowledge, skill, and behavior as a meaningful whole, ie, prescribing practice in its entirety, demonstrates a kind of "tomorrow's knowledge". Students will appreciate and understand the affective, motivational, and cognitive value of the to-be-acquired knowledge before the focus is directed to the actual appropriation and ability to use it. ${ }^{40}$ E-learning has been appreciated for pooling rich factual information that can be accessed by students and referred to by educators throughout the curriculum, which focuses on a specific learning objective or outcome. There is also the opportunity to clearly outline the core learning outcomes to students including knowledge of and understanding about medicines, skills related to prescribing, and attitudes toward drug therapy. ${ }^{25,30,36,38}$

A variety of learning styles are applicable for CPT learning, but in line with the integrated approach, an inquisitive rather than a passive learning method should be adopted. ${ }^{26}$ The problem-based learning style lends itself advantageously to the learning of core CPT, and is highly recommended by the World Health Organization (WHO) Teaching Guide to Good Prescribing, as most prescribing situations are direct attempts to solve a clinical problem regarding the patient, requiring relevant knowledge, skills, and behaviors. ${ }^{26,34,41}$ Also, the theory behind problem-based learning (PBL) is to achieve complex learning, which integrates knowledge, skills, and attitudes, coordinating qualitatively different constituent skills and often transferring what is learnt in didactic lectures or theory-based delivery to real-life problems and settings. $^{42}$

\section{Prescribing is not about the final prescription but the underlying process}

The written or oral prescription of a therapy is where an error manifests itself in a detectable way; however, it is well acknowledged that the complex process of prescribing is, in fact, a high-risk activity within which there are numerous opportunities for inaccuracies and mistakes. ${ }^{3,6,8}$ Aronson describes the formidable task of generating the prescription as demanding a thorough background of pharmacological and therapeutic knowledge and skill; the ability to understand and assess the evidence base, cost effectiveness, and harmbenefit argument; and the facilitative communication skills to discuss management plans for therapy, monitoring, and dosage adjustment with other health care professionals and the patient. ${ }^{25}$ The complexity of this process is compounded by the expanding national formulary, increased prevalence of polypharmacy, higher patient throughput, older and more vulnerable patients, more complex therapeutic regimens, greater demand for information from patients, and the increased threats of litigation. ${ }^{13,26,35,38,43}$ The EQUIP study ${ }^{44}$ provided an insightful synopsis evaluating over 10,000 medication errors, suggesting that most were not a case of simple linear relationships between cause and effect and highlighted the multifaceted nature of the prescribing practice. Reason's Human Error Theory has been utilized to map the range of factors behind poor prescribing at individual, environmental, and organizational levels..$^{13,43}$

Much research has been focused upon designing a model of safe prescribing behavior in order to provide the structure and minimize the potential for error. A recent systematic review found that educational interventions based on the WHO Guide to Good Prescribing have the largest body of evidence to support its use as a promising model for the design of targeted prescribing courses. ${ }^{27}$ Richir et $\mathrm{al}^{33}$ describes the underpinning theory that constituted the basis for this model. The therapeutic decision making or the therapeutic reasoning that precedes and should validate the action of prescribing is described as a process that warrants the investigation and evaluation that has been invested into diagnostic reasoning. Research exists to define the diagnostic reasoning into two subcategories: analytical (system 2), the slower and timeconsuming conscious and systematically based process, and non-analytical (system 1), which is carried out rapidly and subconsciously based on experience and pattern recognition. The argument put forward is that a similar cognitive process takes place when practitioners carry out therapeutic reasoning to arrive at a therapeutic decision, which is transcribed onto a prescription. The subsequent WHO six-step model has been designed as a normative model for therapeutic reasoning and prescribing and is suggested to provide a robust six-step guide to the process of rational and safe prescribing. ${ }^{33}$ Later models developed are fundamentally based on the same principles guiding therapeutic reasoning. The models provide a guide to choose, prescribe, and monitor suitable medicines for an individual patient. ${ }^{45,46}$ Within the WHO model process, the 
recommendation of a core drug list is articulated, which is a store of drugs identified that will be commonly used in clinical practice with the associated prescribing information. The development of this core drug list does not have to be on an individual student basis, but can adopt existing therapeutic pathways and formularies. ${ }^{7,47}$ The cognitive psychology literature would suggest that the role of the core drug list would facilitate and develop a robust system 1 of decision making (non-analytical), which is the fast, intuitive, and reflexive process triggered by pattern recognition, and will make up the bulk of the prescribing workload. Thereby, more time and thinking will be allowed and available for the system 2 process, which is deliberate, slow, and analytical and is required when faced with the prescribing of more unfamiliar drugs. The interrelationship between these two reasoning processes is continuous and dynamic, as further exposure, practice, and learning will allow the conversion of system 2 to system 1 processes, and repeated feedback, reflection, and assessment will be required to review and improve system 1.,33 Bereiter and Scardamalia ${ }^{48}$ describe this process in terms of expertise development that is ongoing and effortful with reflection on situations, and an appropriate equilibrium between automaticity and conscious thought needs to be achieved.

Context learning is recommended to support the development of therapeutic reasoning. The practice of system 1 approach can allow the consolidation of the process whilst also learning the common therapeutic scripts. Fluent recall of information should be facilitated through exposure to the same setting in which it was learnt. Feedback immediately following performance will ensure that correct therapeutic reasoning has been accomplished and thereafter mentally stored. Students should be encouraged to reflect on knowledge and skills to identify and address any deficits or discrepancies. ${ }^{33,49}$ The specific model developed by Lum et $\mathrm{a}^{46}$ share the six steps of the WHO process, but particularly highlight the underpinning requirement for self-reflection on prescribing, seeking, and acting upon constructive feedback and the importance of timely referral. In order for this therapeutic process to be transferrable upon graduation, students should be provided with ample clinical placement provision in which to repetitively put this into practice with real-life problems. Despite the lack of empirical evidence to recommend that clinical placements support this theory, the EQUIP study showed how learning to prescribe is a social process, during which students actively engage with the sociocultural learning environment as they develop cognitive expertise. ${ }^{44}$ Prescribing is a task systemically embedded within the culture and values of the workplace, ${ }^{50}$ making it inappropriate to teach it outside of this context. McLellan et $\mathrm{al}^{29}$ represent this contextual learning in an elegant diagram, which illustrates the elements of a complex skill that are involved in the expert performance of it. The model depicts the integration of knowledge, skill, and attitudes that exist in a continuous feedback loop with the social context. Each time the skill must be practiced in a new setting, learning has to be transferred correctly. Transfer of learning will be hindered and errors are more likely to occur if an integrated approach to addressing the whole practice of prescribing within the social context is not employed. ${ }^{29,49} \mathrm{~A}$ holistic approach to the design of safe prescribing education should therefore be adopted. This will prevent the simplification of the overall complex task to elements that may be easier to transfer to learners in the form of facts and/or practice. Prescribing as a whole has to be acknowledged to be more than the sum of its individual parts. In maintaining this concept, educators will better avoid three persistent problems in the field of education, namely compartmentalization, fragmentation, and the transfer paradox. ${ }^{42}$

Considering the logistics of allowing the continual opportunity to students to learn within context, levels of context have been explored ranging from role-playing with standardized patients in a simulated practice setting to the solution of patient problems in small working groups. ${ }^{33,49}$ There is always emphasis on the improvement of the educational setting so that it is as life-like as possible through the use of real case histories or use of video and laboratory tests rather than written fictional material to ensure that the environment remains high-stress, simulating practice, but with low risk. ${ }^{33}$ The BPS curriculum recommends that pre-prescribing should be in place to allow students to experience the practice where there is less time pressure and better supervision but very close simulation to their expected post-graduation activities. Prescribing simulation has also been suggested that allows rapid scoring and feedback without posing risk to patients. ${ }^{30,37}$ The GMC recommends in its supplementary advice to Tomorrow's Doctors $2009^{51}$ that clinical placements include student assistantships that allow students to put into practice their skills learnt in medical school with real patients under appropriate supervision. This situation would allow students to work in context and carry out clinically skilled tasks, such as prescribing, learn from their clinical team, and prepare better for future practice.

A notable change in the prescribing process is occurring, as evidenced by a recent systematic review on interventions to reduce dosage error, which found that electronic prescribing 
shows considerable promise. ${ }^{52}$ This cannot be ignored, as the adoption of electronic prescribing is becoming more widespread, thereby changing the setting and resources available to the prescribers. Students can be made aware of these resources as a potential facilitator to prescribing, but also appreciate their limitations and currently diverse formats and interfaces that will need to be managed.

\section{Prescribing is not only about personal knowledge, skill, and attitude but it is a participative process}

Culturally, Barber et $\mathrm{al}^{53}$ state that doctors' prescribing is personal to them, with little debate and opportunity for reflective learning. Correction of prescriptions by pharmacists also tends to be isolated discussions with the prescriber, and information is often not shared within the team for consideration and learning. ${ }^{53}$ Newly qualified doctors have described that their experience and observations in practice have heightened their awareness of error. Indeed, it appears that negative examples of prescribing have allowed for reflection and subsequently enhanced sense of professional responsibility and prescribing risk assessment through an explorative and iterative process of behavioral modification. The inward perspective of this process demonstrated no desire to impact external factors, such as environment, colleagues, or systems, and lessons being learnt were not shared, standardized or universal, or resolved at the root cause. ${ }^{6}$ The scientific research carried out by James Reason ${ }^{54,55}$ on medical errors evidences and calls for a significant shift from this person-centered model of error to a systemic approach to error. Concepts of "person" and "individual error" have been replaced by those of "systems" and "safety". ${ }^{56}$ So, despite individual experience of prescribing errors in practice being valuable learning episodes, they have occurred at the potential detriment to patients. Learning has also been only self-directed and usually at an individual perspective. There has been no informing or change to culture or external factors that could improve the interplay of systemic factors that would contribute to a heightened risk awareness and patient-safety culture. ${ }^{6}$ In changing the culture from "person" to "system", this will clarify links between patient safety and interprofessional care. Error disclosure, analysis, and learning have to be recognized as ways to prevent error. Patient safety has to be adopted as a shared responsibility of all members of the team, with team work supported via interpersonal communication, collaboration, coordination, seeking qualified assistance when needed, and accepting colleague supervision, knowledge, and expertise. ${ }^{56,57}$ Interprofessional learning has received much attention as a means to foster this collaborative learning of safe and effective use of medicines between health care professionals. The interactive role of medical, pharmacy, and nursing staff has been demonstrated to enhance safe and effective use of medicines. Interventions at multiple operant factors such as developing a safety-oriented attitude through improving environment conditions, direct staff supervision, and adopting a zero-tolerance policy for incomplete or incorrect prescriptions have been tested in an interprofessional structure..$^{3,32,53}$ In a recent study, it was found that only eight medical schools were, at that time, participating in Interprofessional Education (IPE) but more were in stages of development, and it has been recognized by the BPS as an activity to foster good professional relationships in relation to prescribing. ${ }^{28}$ A one-day conference at Cardiff University School of Medicine allowed schools across the UK to present findings of their recent IPE activities, which reflected the value students attributed to the experience with respect to confidence in their own and other health care professionals' role; increased awareness of prescribing resources, including members of the health care team; and appreciation of the knowledge base of each profession and therefore more understanding in error causation. ${ }^{58}$ These activities show the uptake and motivation to act upon the IPE agenda, but as yet little empirical evidence exists to support and inform what IPE should look like to achieve outcomes in medication safety and safe prescribing practice. A report by a final-year medical student showed how an innovative learning experience that involved the checking of drug charts on the post-take ward round allowed the identification and addressing of errors to ensure that they were vocalized and rectified. ${ }^{59}$ The activity resembles that of a ward pharmacist, and as such highlighted that the consideration of prescribing from the perspective of another health care professional offers valuable education about common errors and the importance of an open, noblame culture in addressing them. One significant barrier to effective reflection and discussion of errors in practice was observed to be the attitude and approachability of the leading consultant on the ward. ${ }^{59}$ In order to manage this potentially limiting factor, the implementation of "nearpeer" teaching and tutorials have been trialed and tested ${ }^{60}$ and recommended by the BPS. ${ }^{37}$ Both students and junior doctors report improved prescribing confidence. The process also appears to harness enthusiasm to participate in future teaching activities and nurtures the open debate required around the discussion and awareness of error. Students express preference for delivery of training by junior doctors due to the ease to identify and approach, and tutors claim 
that the teaching provides an appreciated chance to reflect on practice, recognize, and address deficits in knowledge, which thereby aid in their continuing development as prescribers. ${ }^{60}$ However, it is worth noting that this strategy paradoxically recommends teaching of students by those practitioners that are demonstrating the poorest prescribing in practice. The profound effect of role-modeling on professional behaviors of students is well acknowledged. Therefore, formal development programs for junior doctors contributing to prescribing teaching should be a prerequisite. This training should foster excellence in doctor role-modeling and ensure that safe and effective practice is communicated. ${ }^{61}$

\section{Prescribing should not be presumed, it should be appropriately proven}

Qualification of a doctor includes a license to prescribe due to the assumption that all GMC outcomes have been assessed and successfully achieved; this includes the specific outcome:

"prescribe drugs safely, effectively, and economically". ${ }^{2}$

However, due to the ambiguity of this outcome, which covers a practice that is so multifaceted and requires the demonstration of knowledge, skills, and behavior, there is significant variation in methods of assessment employed across medical schools within the UK. ${ }^{36}$ It is subsequently difficult to ascertain the level of competence that is being achieved via respective curricula. ${ }^{16}$ Heaton et al ${ }^{16}$ highlighted the generic terms used to describe the required exit competencies for graduates that have made the design of appropriate assessments very arbitrary and problematic, and found a majority of surveyed medical students and Foundation Year 1 (FY1) doctors felt that they had not been thoroughly assessed in prescribing. ${ }^{16}$ Miller's triangle of clinical assessment has been used to model the evidencing of competence development. It depicts the hierarchical levels of assessing clinical skill, namely knowledge (knows), competence (knows how), performance (shows how), and action (does). ${ }^{33}$ However, it has been subsequently highlighted that clinical performance will be impacted by many factors such as time availability, tiredness, stress, etc that distorts the "does" that is assessed in a controlled environment. ${ }^{62}$ To encompass these external and internal factors and relate competence to performance, Rethans et $\mathrm{al}^{23}$ proposed modifications to Miller's triangle and developed "The Cambridge model". This model attests the need to assess global competence or performance of doctors in training in as realistic a way as possible. The Objective Structured Clinical Examination (OSCE) and structured clinical examinations are well recognized methods for assessing at the peak of Miller's pyramid: competence and performing, with proven use in assessing for therapeutic competence as a whole, rather than on assessing the separate components. ${ }^{30,33,63}$ The validity of the OSCE or other clinical assessment tools is dependent upon the number of stations and skills evaluated to give a coherent view of the students' clinical skill level. The need to assess the students in a manner similar to that in which they were taught has also been recognized and links back to the principles of context learning. The exposure to a setting similar to that of the learning environment will facilitate the recall of acquired knowledge and skills and the therapeutic reasoning that underpins its appropriate application. ${ }^{33}$ The BPS promotes the employment of the OSCE as an ideal format of assessment acknowledging that written questions are useful for assessing the underlying knowledge but inadequate as a measure of prescribing skill. Ross and Loke ${ }^{27}$ found the inadequacy of assessing prescribing by a single OSCE station due to the complexity of the task and need to assess all separate components. ${ }^{27}$ However, caution must be applied that the components are not individually assessed since it is the amalgamation of them that reflects the complexity and context of real-life practice..$^{29}$ Maxwell $^{37}$ lists skills such as the writing

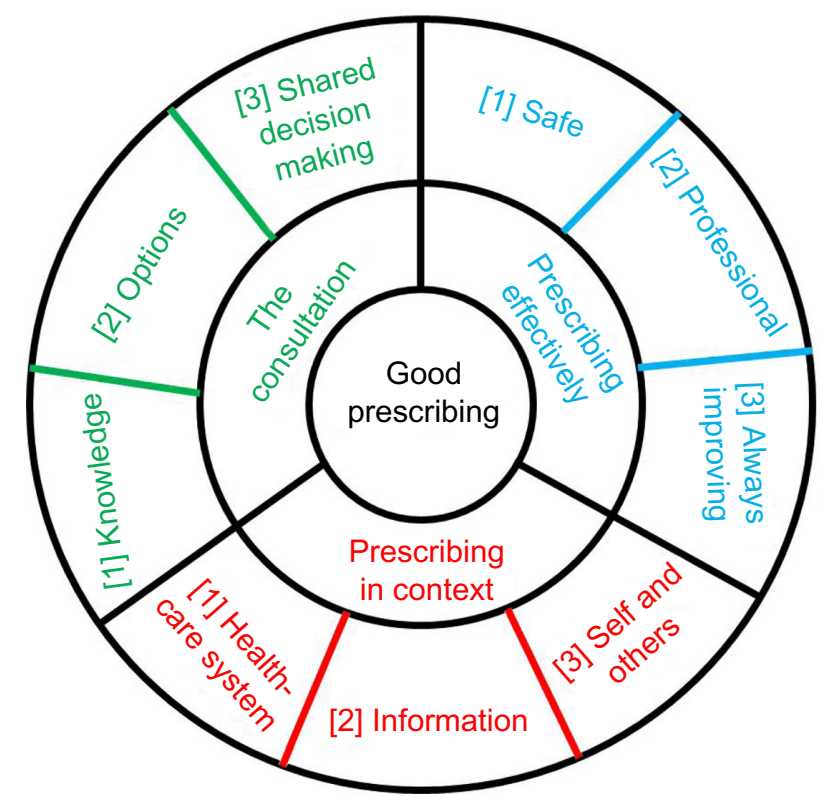

Figure I The three domains of good prescribing, as depicted by the NPC competency framework: Prescribing in context (red); The consultation (green) and Prescribing effectively (blue), all contain three dimensions of competency ([I], [2], and [3]) within which are statements which describe an activity or outcome a prescriber should be able to demonstrate.

Notes: This figure provides a color coded key to show how these activities/ outcomes have been mapped onto the outcomes from GMC Tomorrow's Doctors 2009 in Table I. Data from National Prescribing Centre ${ }^{21}$ and from the General Medical Council. ${ }^{2}$

Abbreviation: NPC, National Prescribing Centre. 

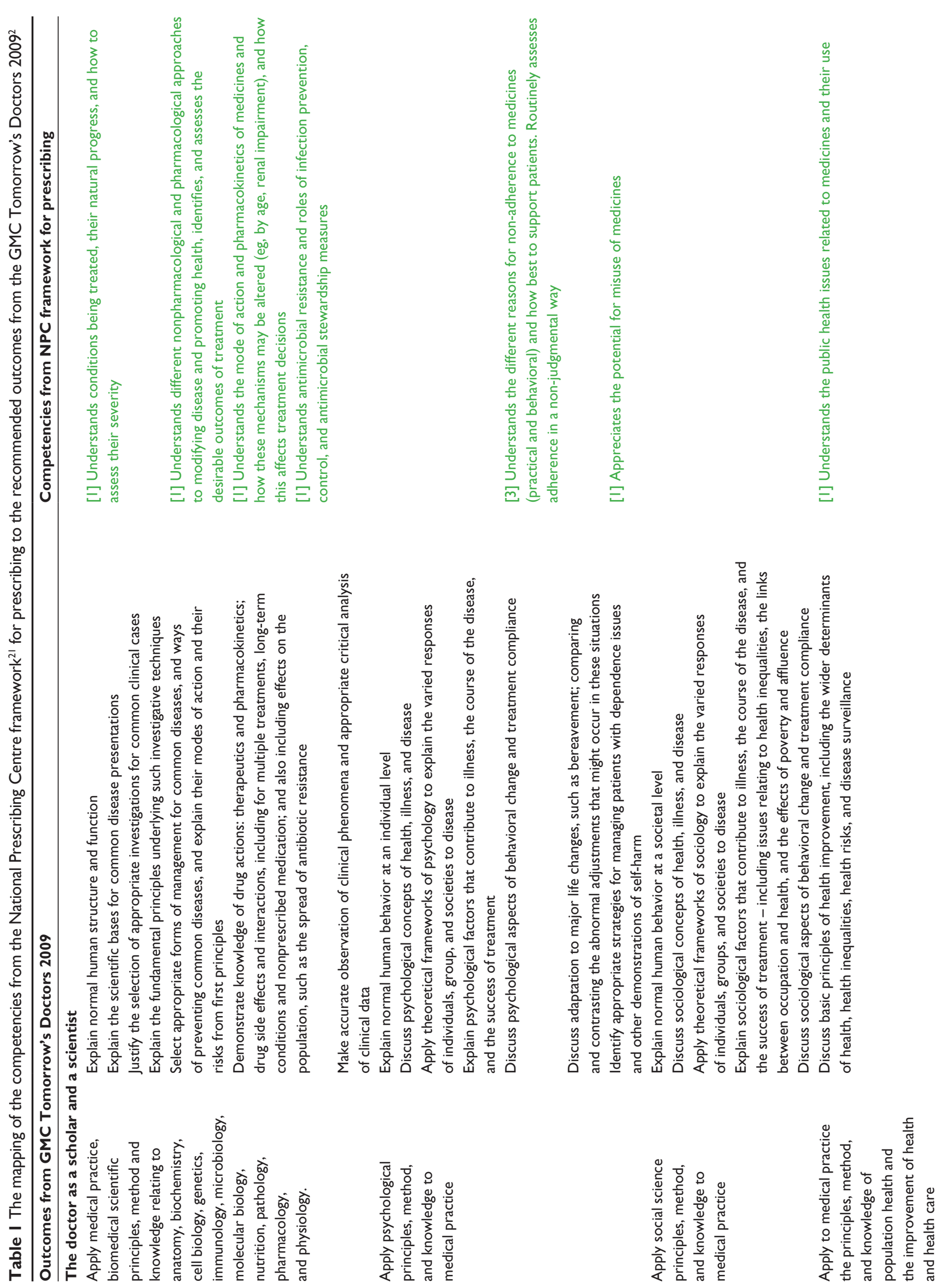

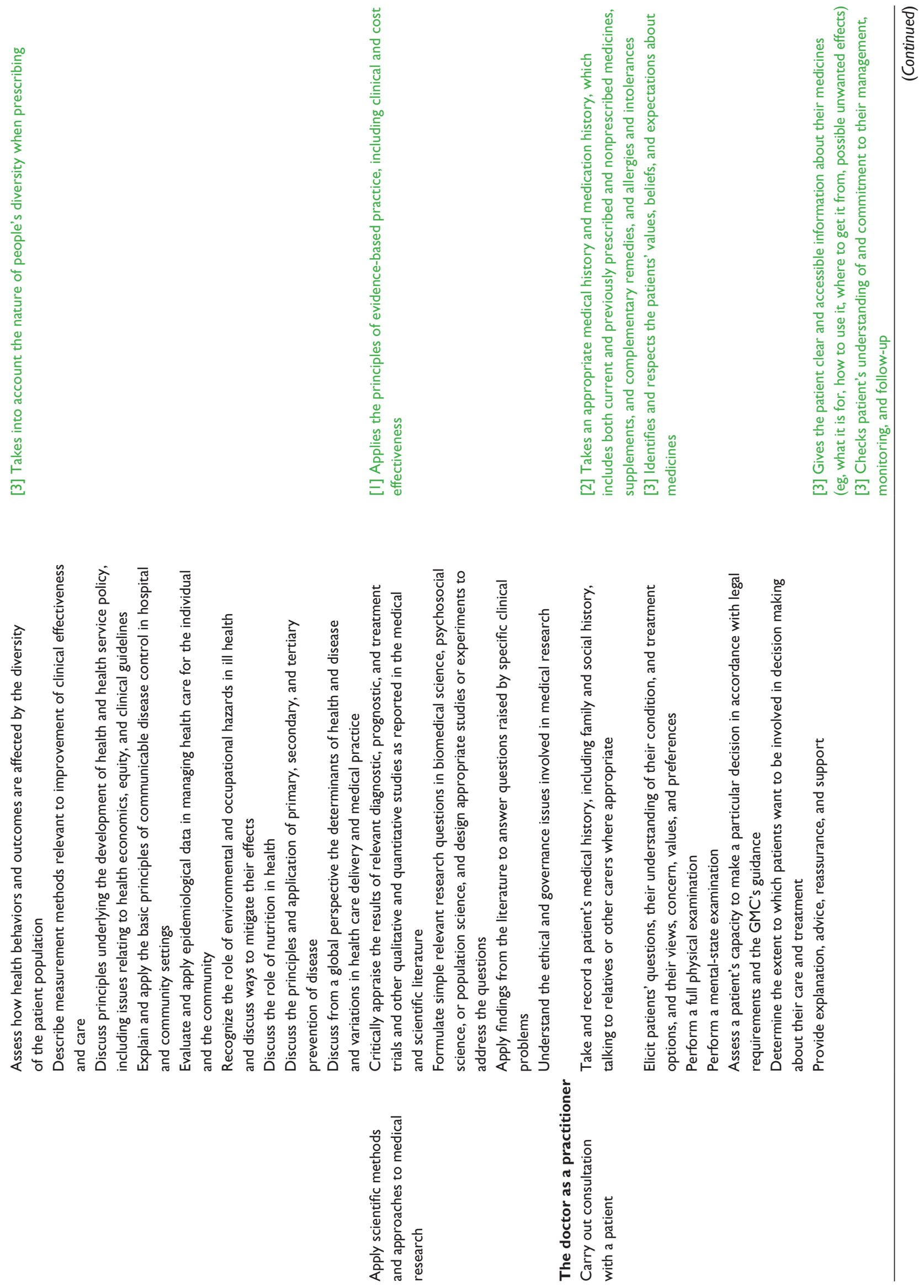

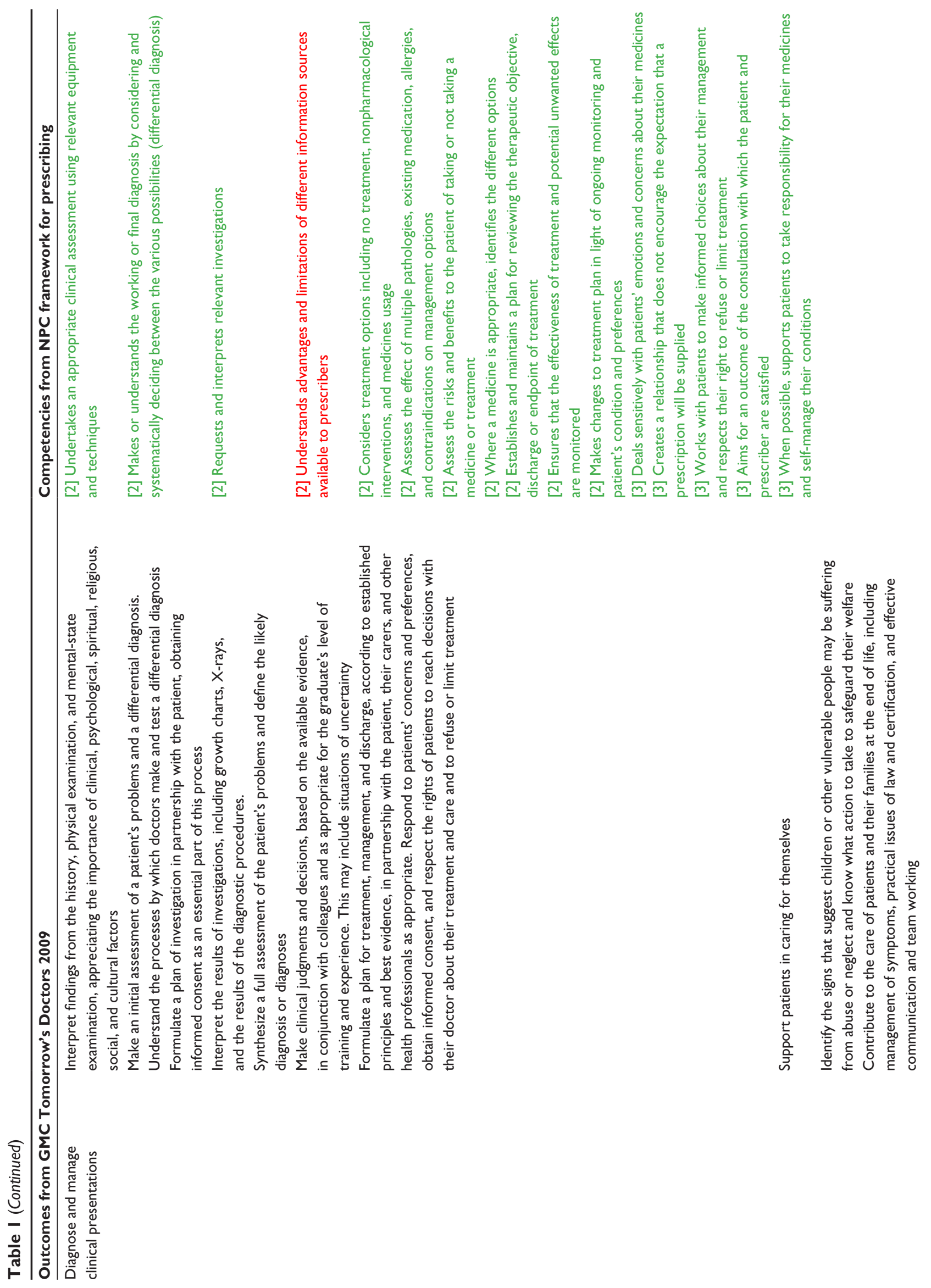


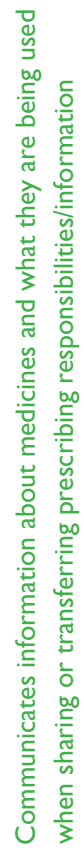

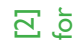

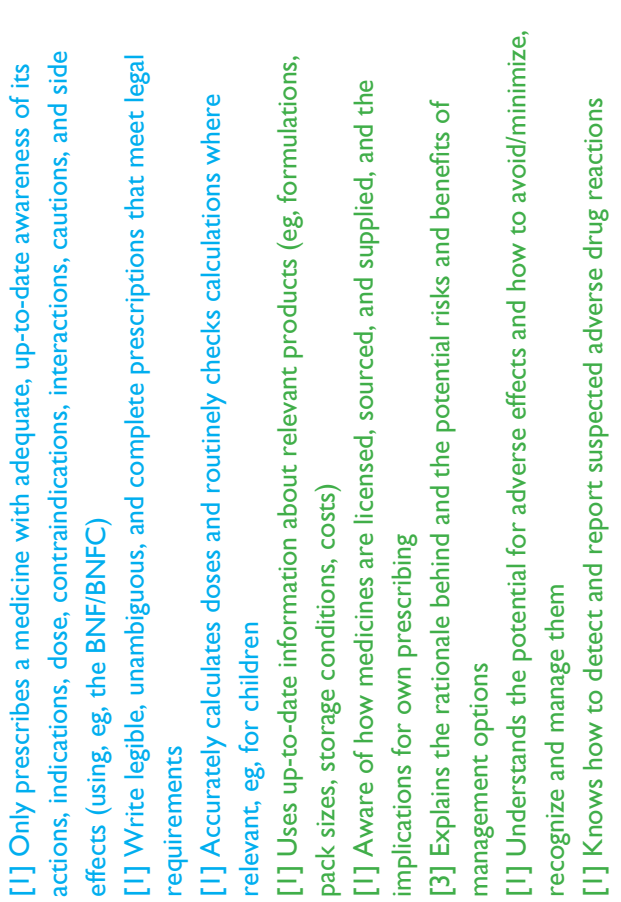

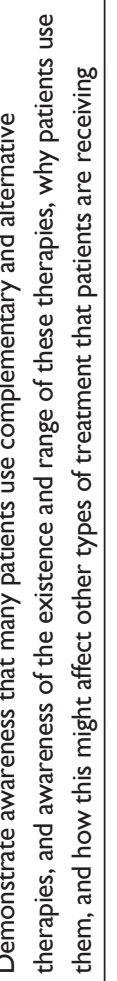

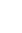
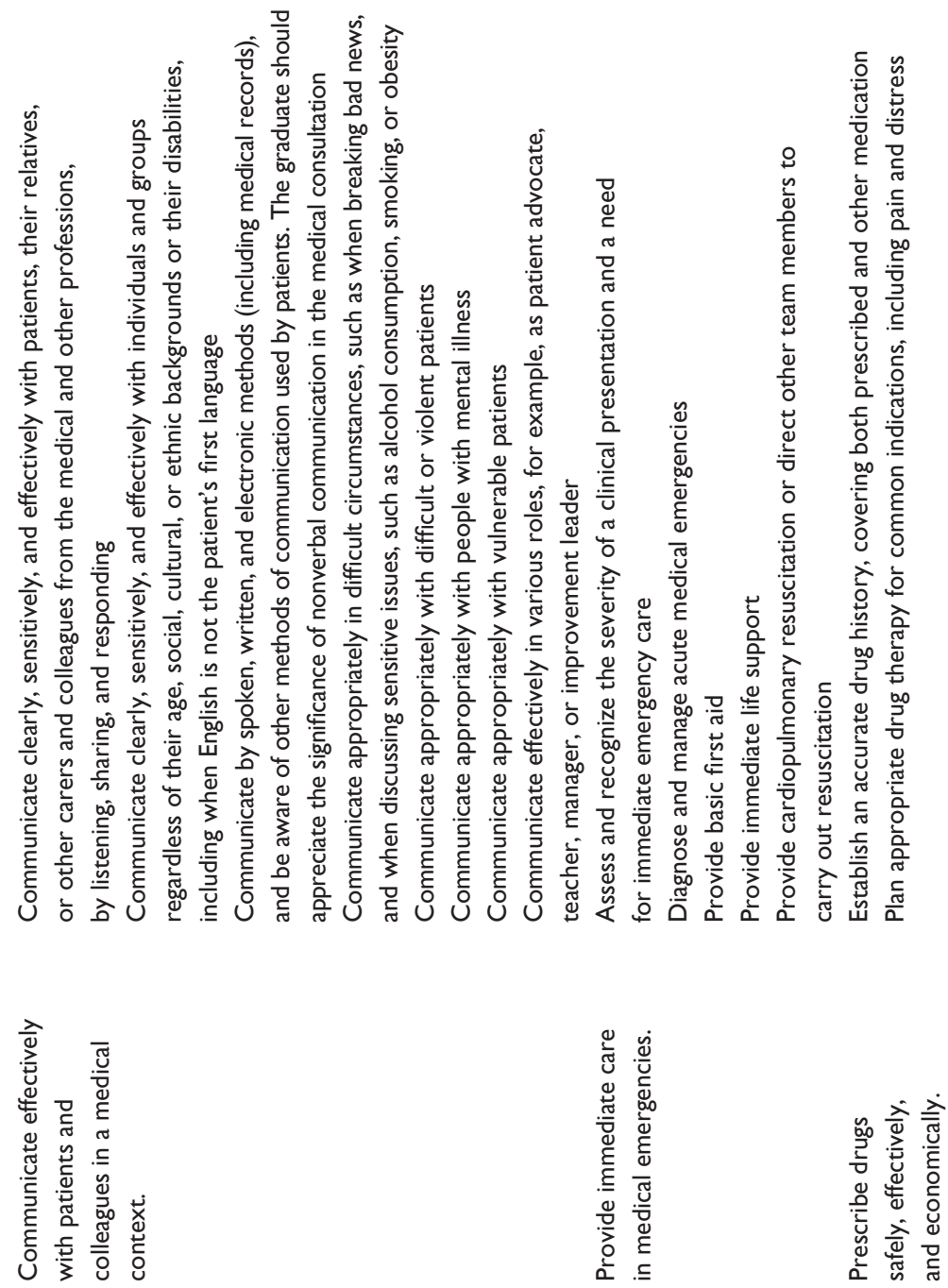


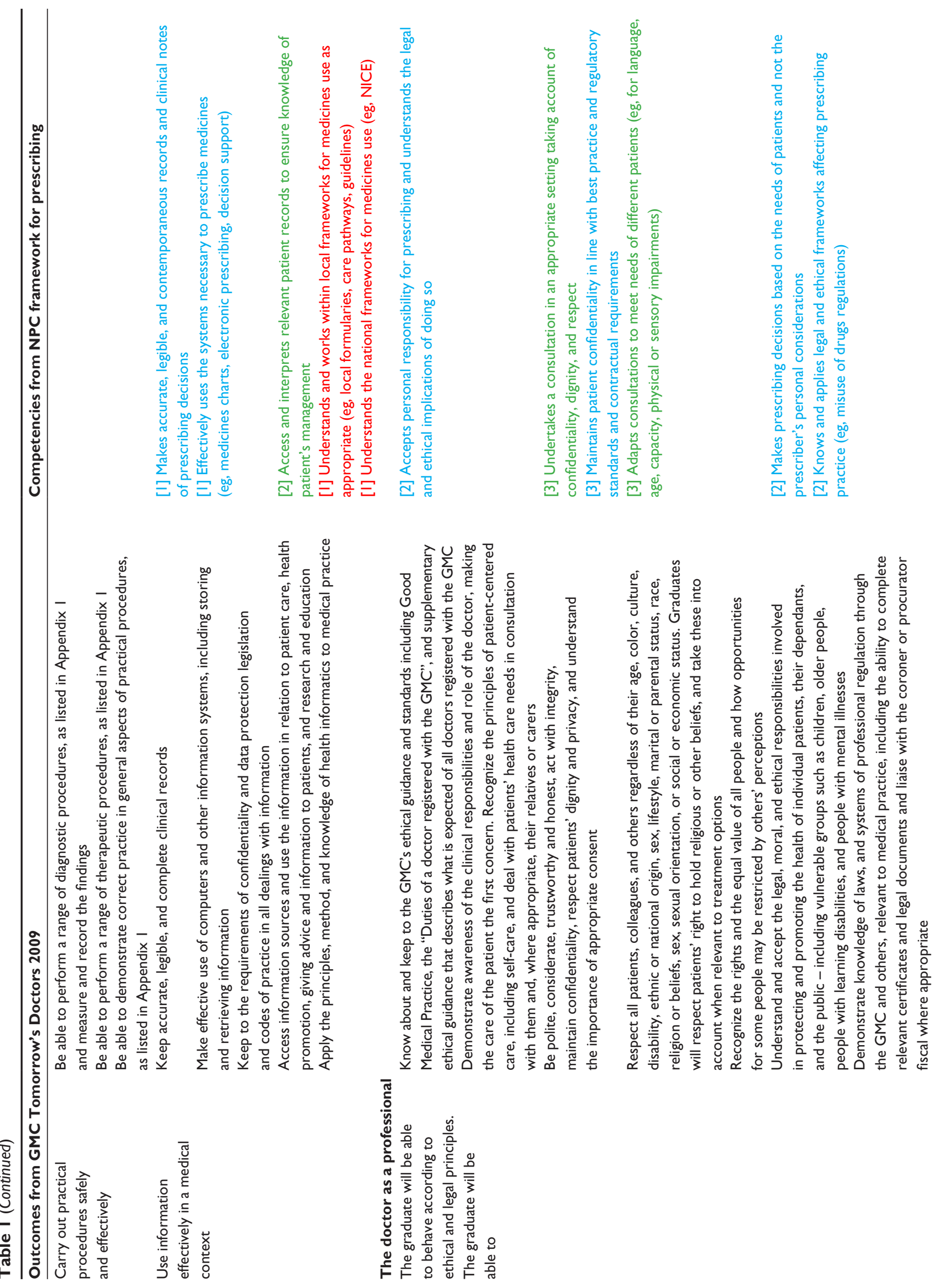



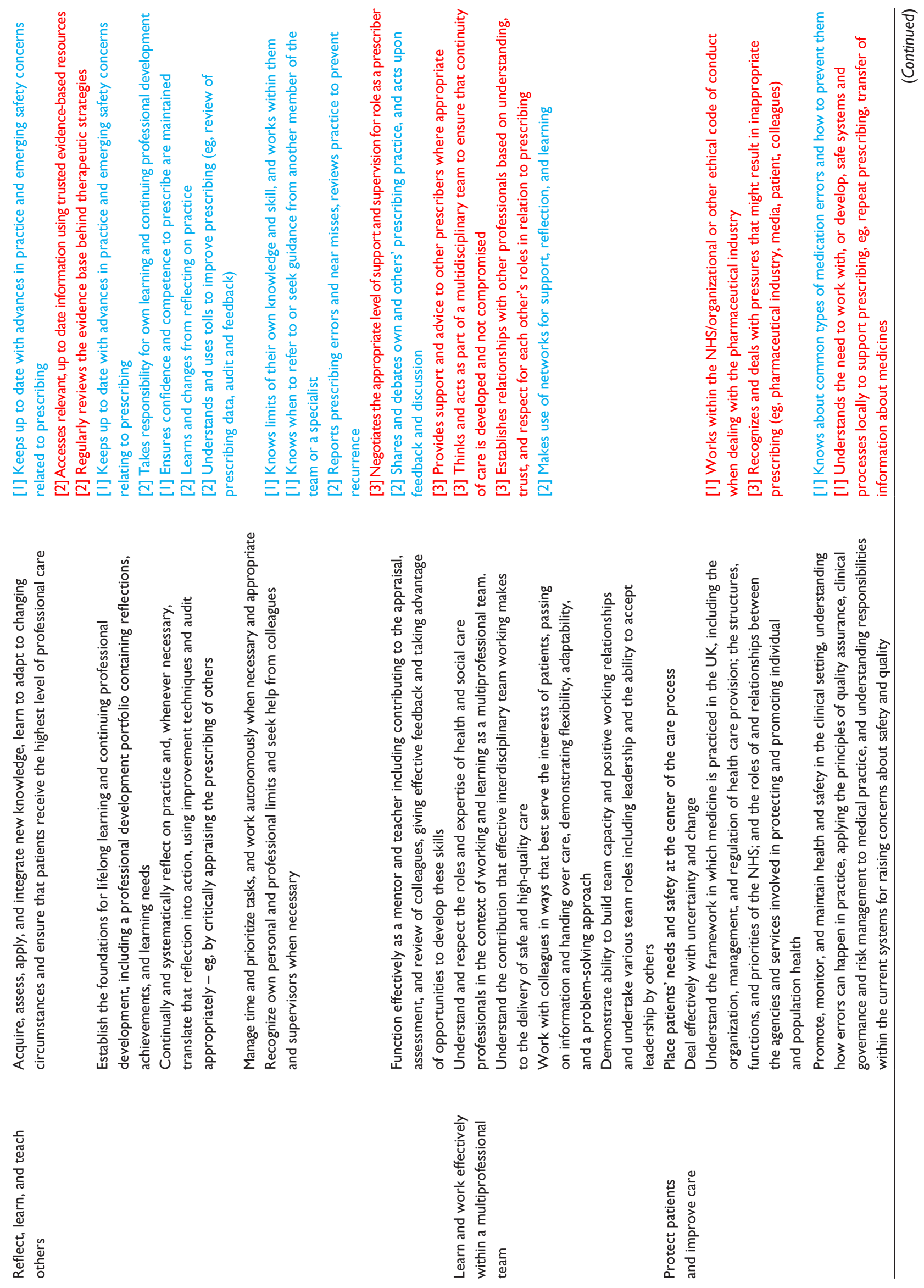
of a prescription, offering information to a patient about a medicine, and identifying potentially hazardous prescriptions as some of the skills that make up the prescribing practice that warrant assessment. ${ }^{37}$ The most challenging facet of clinical competence to examine is level 4 of Miller's triangle, which relates specifically to performance, factoring in the additional system and individual-related influences from the modified Cambridge model. In order to ensure effective assessment, it is crucial to directly observe students in practice. ${ }^{62}$ Lum and Coombe ${ }^{46}$ reflect that direct observation is required to assess for level 4 "does". They elaborate that direct observation of procedural skill is required so as to prove competence in surgical procedures, and so an equal requirement should exist for prescribing. ${ }^{46} \mathrm{~A}$ system of observing a collection of videotaped consultations has been employed to assess a trainee's clinical skills. Standardized patients have also provided an opportunity for patient-simulated observations, but caution is advised in the training of the patients, validity of patient cases, and scoring scales. Another method assesses attitude via the use of structured or open questionnaires completed by members of the health care team to inform a $360^{\circ}$ appraisal for medical staff. Personal logs and portfolios are potentially also useful for attitude assessment. ${ }^{62}$ The difficulties of these methods tie in with the question of where in the undergraduate-postgraduate continuum is prescribing best taught and assessed. Potentially, the undergraduate setting is sufficient to teach and assess up to and including level 3 of Miller's triangle. The complete amalgamation and assessment of knowledge, skill, and behavior cannot be fully facilitated in an environment that does not offer the additional exposure to individual and systemic influences, and therefore it is better situated in the clinical setting. Indeed, the recent GMC document proposing the development and implementation of a National Licensing Examination (NLE) could offer the opportunity to assess graduates after a dedicated time in practice (eg, as suggested at the end of FY1). The initiative also intends to combine a discrete Prescribing Safety Assessment (PSA) that can be designed as such to standardize the process of assessment. The recent GMC Good Medical Practice guidance, ${ }^{64}$ which refers specifically to prescribing and managing medicines and devices, could be used to frame the expected behaviors and competences of qualified doctors in clinical practice and thereby inform the development of this PSA. The case for an NLE incorporating a PSA presented by the GMC argues for the objective assurance of a common standard of practitioners to help protect patients and also brings consistency to the assessment of outcomes of all graduates irrespective of medical school. ${ }^{65}$ 
In considering what could be achievable in the undergraduate curriculum, the NPC single-competency framework for prescribers could offer more objective definitive criteria to clarify and expand upon the GMC's general learning outcomes. This framework is a highly detailed depiction of the components that make up the prescribing process and provides an outline of competencies that, if acquired and maintained, can aid prescribers to become and remain effective and safe in their area of practice. ${ }^{21}$ The competencies have been mapped to the GMC outcomes based on subjective judgment (Table 1 and Figure 1 ), which is open to debate by other medical educators or curriculum designers. However, we retain the belief that the competencies can be accommodated even if not in our designated loci. This exercise demonstrates that, by absorbing the competency framework, educators can review their current curriculum that would have been designed to meet the GMC outcomes and visualize where competencies relating specifically to prescribing can be taught, learnt, and assessed. We speculate that existing material can be adjusted to add a dimension relating to prescribing and thereby offer opportunities for teaching, learning, and assessment to be integrated or designed to include this element. It is important to highlight that in any integrated assessment, there should be an identifiable and robust component devoted to the knowledge and skills that support drug prescribing, and it should not be "compensatable" by performance in other areas. ${ }^{26,37}$ This would ensure prescribing is a practice that receives its due attention and continual reference vertically and horizontally throughout the medical curriculum. A further layer to add to this mapping to aid educators and curriculum designers would be the addition of evidencebased robust assessment strategies against each individual or collection of competencies that have been trialed and tested for validity, generalizability, and vigor. This sharing of best practice amongst medical schools will truly allow the pedagogical challenge of the teaching and assessing of prescribing to be realized and overcome in the most cohesive and collaborative way.

\section{Summary}

The complexity of the practice of prescribing needs to be acknowledged and examined before one can be confident in its teaching to a safe and effective level. Also, this appreciation of intricacy of the role as a whole should be clear and visible to the undergraduate student so as to guide and frame the fostering of appropriate knowledge, attitudes, and behaviors toward this learning outcome. We have outlined and examined some of the component subsystems around the teaching, learning, and assessment of the system of prescribing within the undergraduate medical curriculum. The discussion of our findings is our "worldview", in line with systems thinking, on how to teach safe and effective prescribing. The themes offer the opportunity for reflection and potential resolutions to identified issues with some current educational concepts. Finally, the identified detailed framework that can be subsumed onto the GMC learning outcomes demonstrates that nationally commissioned recommendations and guidelines do exist that can inform and add further objective, standardized structure to educational curriculum.

\section{Disclosure}

The authors report no conflicts of interest in this work.

\section{References}

1. Goodreads.com [homepage on the Internet]. Richard Diaz Quotes; 2014 Available from: https://www.goodreads.com/author/quotes/2490467. Richard_Diaz. Accessed December 8, 2014.

2. General Medical Council. Tomorrow's Doctors: Outcomes and Standards for Undergraduate Medical Education. London: General Medical Council; 2009.

3. Coombes I, Mitchell C, Stowasser D. Safe medication practice tutorials: a practical approach to preparing prescribers. Clin Teach. 2007;4(3): 128-134.

4. Patricio KP, Borges Alves NA, Arenales NG, Queluz TT. Teaching the rational use of medicines to medical students: a qualitative research. BMC Med Educ. 2012;19:12.

5. Gwee MCE. Teaching of medical pharmacology: the need to nurture the early development of desired attitudes for safe and rational drug prescribing. Med Teach. 2009;31(9):847-854.

6. Gordon M, Catchpole K, Baker P. Human factors perspective on the prescribing behavior of recent medical graduates: implications for educators. Adv Med Educ Pract. 2013;4:1-9.

7. Baker E, Roberts AP, Wilde K, et al. Development of a core drug list towards improving prescribing education and reducing errors in the UK. Br J Clin Pharmacol. 2011;71(2):190-198.

8. Celebi N, Weyrich P, Riessen R, Kirchhoff K, LammerdingKoeppel M. Problem-based training for medical students reduces common prescription errors: a randomised controlled trial. Med Educ. 2009;43(10):1010-1018.

9. McCarthy RM, Hilmer SN. Teaching Junior Medical Officers safe and effective prescribing. Intern Med J. 2013;43(11):1250-1253.

10. Tully MP, Ashcroft DM, Dornan T, Lewis PJ, Taylor D, Wass V. The causes of and factors associated with prescribing errors in hospital inpatients: a systematic review. Drug Saf. 2009;32(10):819-836.

11. Rothwell C, Burford B, Morrison J, et al. Junior doctors prescribing: enhancing their learning in practice. Br J Clin Pharmacol. 2012;73(2): 194-202.

12. Illing J, Morrow G, Kergon C, et al. How Prepared are Medical Graduates to Begin Practice? London: General Medical Council; 2008.

13. Baldwin M, Abouyannis M, Butt T. Essential therapeutics skills required of junior doctors. Perspect Med Educ. 2012;1(5-6):225-236.

14. Sandilands EA, Reid K, Shaw L, et al. Impact of a focussed teaching programme on practical prescribing skills among final year medical students. Br J Clin Pharmacol. 2011;71(1):29-33.

15. Tobaiqy M, McLay J, Ross S. Foundation year 1 doctors and clinical pharmacology and therapeutics teaching. A retrospective view in light of experience. Br J Clin Pharmacol. 2007;64(3):363-372. 
16. Heaton A, Webb DJ, Maxwell SRJ. Undergraduate preparation for prescribing: the views of 2413 UK medical students and recent graduates. Br J Clinl Pharmacol. 2008;66(1):128-134.

17. White $\mathrm{C}$. Foundation trainees could face prescribing test. BMJ Careers. 2013. Available from: http://careers.bmj.com/careers/advice/viewarticle.html?id=20015122. Accessed December 8, 2014.

18. Conroy S, North C, Fox T, et al. Educational interventions to reduce prescribing errors. Arch Dis Child. 2008;93(4):313-315.

19. Scobie SD, Lawson M, Cavell G, Taylor K, Jackson SHD, Roberts TE. Meeting the challenge of prescribing and administering medicines safely: structured teaching and assessment for final year medical students. Med Educ. 2003;37(5):434-437.

20. Kozer E, Scolnik D, Macpherson A, Rauchwerger D, Koren G. The effect of a short tutorial on the incidence of prescribing errors in pediatric emergency care. Can J Clin Pharmacol. 2006;13(3):e285-e291.

21. National Prescribing Centre. A Single Competency Framework for all Prescribers. Liverpool: National Institute for Health and Clinical Excellence; 2012.

22. Whiddett SHS. The Competencies Handbook. London: Institute of Personnel and Development; 1999.

23. Rethans JJ, Norcini JJ, Barón-Maldonado M, et al. The relationship between competence and performance: implications for assessing practice performance. Med Educ. 2002;36(10):901-909.

24. Reynolds M, Holwell S. Introducing Systems Approaches. Systems Approaches to Managing Change: A Practical Guide. New York: Springer; 2010:1-23.

25. Aronson JK, Henderson G, Webb DJ, Rawlins MD. A prescription for better prescribing. Br J Clin Pharmacol. 2006;333(7566):459-460.

26. Maxwell S, Walley T. Teaching safe and effective prescribing in UK medical schools: a core curriculum for tomorrow's doctors. Br J Clin Pharmacol. 2003;55(6):496-503.

27. Ross S, Loke YK. Do educational interventions improve prescribing by medical students and junior doctors? A systematic review. Br J Clin Pharmacol. 2009;67(6):662-670.

28. O’Shaughnessy L, Haq I, Maxwell S, Llewelyn M. Teaching of clinical pharmacology and therapeutics in UK medical schools: current status in 2009. Br J Clin Pharmacol. 2010;70(1):143-148.

29. McLellan L, Tully MP, Dornan T. How could undergraduate education prepare new graduates to be safer prescribers? Br J Clin Pharmacol. 2012;74(4):605-613.

30. Ross S, Maxwell S. Prescribing and the core curriculum for tomorrow's doctors: BPS curriculum in clinical pharmacology and prescribing for medical students. Br J Clin Pharmacol. 2012;74(4):644-661.

31. Harding S, Britten N, Bristow D. The performance of junior doctors in applying clinical pharmacology knowledge and prescribing skills to standardized clinical cases. Br J Clin Pharmacol. 2010;69(6):598-606.

32. Kamarudin G, Penm J, Chaar B, Moles R. Educational interventions to improve prescribing competency: a systematic review. BMJ Open. 2013;3(8):e003291.

33. Richir MC, Tichelaar J, Geijteman ECT, de Vries TPGM. Teaching clinical pharmacology and therapeutics with an emphasis on the therapeutic reasoning of undergraduate medical students. Eur J Clin Pharmacol. 2008;64(2):217-224.

34. Vollebregt JA, Metz JCM, de Haan M, Richir MC, Hugtenburg JG, de Vries T. Curriculum development in pharmacotherapy: testing the ability of preclinical medical students to learn therapeutic problem solving in a randomized controlled trial. Br J Clin Pharmacol. 2006;61(3): $345-351$.

35. George CJ, Jacobs LG. Geriatrics medication management rounds: a novel approach to teaching rational prescribing with the use of the medication screening questionnaire. J Am Geriatr Soc. 2011;59(1): $138-142$.

36. Likic R, Maxwell SRJ. Prevention of medication errors: teaching and training. Br J Clin Pharmacol. 2009;67(6):656-661.

37. Maxwell SRJ. How should teaching of undergraduates in clinical pharmacology and therapeutics be delivered and assessed? Br J Clin Pharmacol. 2012;73(6):893-899.
38. Maxwell SRJ, McQueen DS, Ellaway R. eDrug: a dynamic interactive electronic drug formulary for medical students. Br J Clin Pharmacol. 2006;62(6):673-681.

39. Galperin G. Intellectual capabilities among older preschool children: on the problem of training and development. In: WW Hartup, editor. Review of Child Development Research. Vol. 6. Chicago: University of Chicago Press; 1982:526-546.

40. Kozulin A. Vygotsky's Educational Theory in Cultural Context. Cambridge: Cambridge University Press; 2003.

41. Scordo KA. Teaching students about the WHO guide to good prescribing. Nurse Pract. 2014;39(3):51-54.

42. Van Merriënboer JJ, Kirschner PA. Ten Steps to Complex Learning: A Systematic Approach to Four-Component Instructional Design. Routledge, London; 2012.

43. Ross S, Loke YK. Development of learning outcomes for an undergraduate prescribing curriculum (British Pharmacological Society prescribing initiative). Br J Clin Pharmacol. 2010;70(4):604-608.

44. Dornan T, Ashcroft D, Heathfield H, et al. An in-depth investigation into causes of prescribing errors by foundation trainees in relation to their medical education. EQUIP Study. Final report to the General Medical Council, University of Manchester: School of Pharmacy and Pharmaceutical Medicine; 2009. Available from: http://psnet.ahrq.gov/ resource.aspx?resourceID=17269. Accessed October 14, 2014.

45. Iglar K, Kennie N, Bajcar J. I can prescribe a drug: mnemonic-based teaching of rational prescribing. Fam Med. 2007;39(4):236-240.

46. Lum EMC, Mitchell C, Coombes I. The competent prescriber: 12 core competencies for safe prescribing. Aust Prescr. 2013;36:13-16.

47. De Vries TP, Daniels JM, Mulder CW, et al. Should medical students learn to develop a personal formulary? Eur J Clin Pharmacol. 2008; 64(6):641-646.

48. Bereiter CS, Scardamalia M. Surpassing Ourselves: An Inquiry into the Nature and Implications of Expertise. Chicago: Open Court Publishing Company; 1993.

49. Richir MC, Tichelaar J, Stanm F, et al. A context-learning pharmacotherapy program for preclinical medical students leads to more rational drug prescribing during their clinical clerkship in internal medicine. Clin Pharmacol Ther. 2008;84(4):513-516.

50. Kilminster S, Zukas M, Quinton N, Roberts T. Learning practice? Exploring the links between transitions and medical performance. J Health Organ Manag. 2010;24(6):556-570.

51. General Medical Council. Clinical Placements for Medical Students: Advice Supplementary to Tomorrow's Doctors. London: 2009.

52. Conroy S, Sweis D, Planner C, et al. Interventions to reduce dosing errors in children: a systematic review of the literature. Drug Saf. 2007; 30(12):1111-1125.

53. Barber N, Rawlins M, Franklin BD. Reducing prescribing error: competence, control, and culture. Qual Saf Health Care. 2003;12:I29-I32.

54. Reason J. Human Error. New York: Cambridge University Press; 1990.

55. Reason J. Human error: models and management. BMJ. 2000; 320(7237):768-770.

56. Infante C. Bridging the "system's" gap between interprofessional care and patient safety: sociological insights. J Interprof Care. 2006;20(5): $517-525$.

57. Reason J. Beyond the organisational accident: the need for "error wisdom" on the frontline. Qual Saf Health Care. 2004;13(Suppl 2): ii28-ii33.

58. Bradley P, Golaup C, John D, Routledge PA. Learning and Working Together to Improve Safety through Better Prescribing. Interprofessional Education Conference. Wales: Cardiff University; 2013.

59. Conroy-Smith E, Herring R, Caldwell G. Learning safe prescribing during post-take ward rounds. Clin Teach. 2011;8(2):75-78.

60. Gibson KR, Qureshi ZU, Ross MT, Maxwell SR. Junior doctor-led 'near-peer' prescribing education for medical students. Br J Clin Pharmacol. 2014;77(1):122-129.

61. Nazar M, Kendall K, Day L, Nazar H. Decolonising medical curricula through diversity education: lessons from students. Med Teach. 2014:1-9. In press 2014. 
62. Carr S. Assessing clinical competency in medical senior house officers: how and why should we do it? Postgrad Med J. 2004;80(940):63-66.

63. van Lohuizen MT, Kuks JBM, van Hell EA, Raat AN, Cohen-Schotanus J. Learning strategies during clerkships and their effects on clinical performance. Med Teach. 2009;31(11):E494-E499.

64. General Medical Council. Good Practice in Prescribing and Managing Medicines and Devices. London: 2013.
65. General Medical Council. Item to Consider: National Licensing Examination; 2014. Available from: http://www.gmcuk.org/06 National_Licensing_Examination.pdf_57876215.pdf. Accessed November 12, 2014.

\section{Publish your work in this journal}

Advances in Medical Education and Practice is an international, peerreviewed, open access journal that aims to present and publish research on Medical Education covering medical, dental, nursing and allied health care professional education. The journal covers undergraduate education, postgraduate training and continuing medical education including emerging trends and innovative models linking education, research, and health care services. The manuscript management system is completely online and includes a very quick and fair peer-review system. Visit http://www.dovepress.com/testimonials.php to read real quotes from published authors.

Submit your manuscript here: http://www.dovepress.com/advances-in-medical-education-and-practice-journal 